\title{
Uplink Sum Rate and Capacity of Hybrid Precoding mmWave Massive MIMO System
}

\author{
Vikash Sachan*, Ritesh Kumar Mishra \\ Department of Electronics and Communication Engineering, National Institute of Technology Patna, India
}

Corresponding Author Email: vikash.ece15@nitp.ac.in

https://doi.org/10.18280/ts.360205

Received: 15 January 2019

Accepted: 3 April 2019

\section{Keywords:}

MIMO, massive MIMO, millimeter wave, hybrid precoding and combining

\begin{abstract}
Massive multiple input multiple output (MIMO) systems offer an improvement in the uplink sum rate with increasing the number of base station antennas. Massive MIMO system needs the perfect channel state information (perfect CSI) and imperfect channel state information (imperfect CSI) for deriving the achievable sum rate. The Uplink sum rate is derived for the $\mathrm{ZF}$ receiver and the $\mathrm{MRC}$ receiver with perfect CSI and imperfect CSI. The zero forcing $(\mathrm{ZF})$ receiver outperforms maximal ratio combining (MRC) receiver. With imperfect CSI as the number of users increases the uplink sum rate also increases. The channel capacity is derived for the Millimeter wave (mmWave) MIMO system employing MMSE receiver. The mmWave massive MIMO system requires a large number of radio frequency (RF) chains where as the number of the RF chains increases the capacity of the system also improves.
\end{abstract}

\section{INTRODUCTION}

Current wireless networks operate at the carrier frequency below $6 \mathrm{GHz}$. The mmWave massive MIMO system works beyond $6 \mathrm{GHz}$. The massive MIMO system at mmWave range gives the benefits of a large bandwidth without compromising on device size and therefore it is facilitating the $5 \mathrm{G}$ technology. The mmWave massive MIMO works beyond $6 \mathrm{GHz}$ [1-2]. Due to the large available bandwidth of the mmWave frequencies, high transmission rates can be achieved. IEEE 802.11 ad-WLAN standard based on the mmWave provides data rates of $6 \mathrm{Gbps}$ at frequencies around $60 \mathrm{GHz}$. But there are several problems to implement these types of systems due to rain attenuation, atmospheric absorption and severe path loss [3]. However, small chips are having huge number of antenna arrays at frequencies beyond $6 \mathrm{GHz}$ and can provide high received signal power [4]. Analysis of systems with and without channel state information has been done [5-8]. There is a great deal of interest in the multiuser multiple input multiple output (MU-MIMO) containing a high number of antennas in a base station (BS). High number of MU-MIMO systems are serving hundreds of users by providing hundreds of antenna arrays. The design and analysis of MU-MIMO systems is appealing substantial interest in the latest research areas [9-11]. Small scale fading can be averaged out due to high antenna array. The cost of each antenna is low because the real size of the antenna is small. As the number of BS antenna increases, the random channel vectors become orthogonal between $\mathrm{BS}$ and the users. One important advantage of the large MIMO system is that there is a reduction in the transmitting power. In the uplink, decreasing the transmit power of the node will result in improving the battery life. In the downlink, the large amount of power consumed by the base stations is exhausted by the power amplifiers, associated circuits and cooling circuits [12-14]. Wireless backhaul is challenging in closely dispersed small cells in urban environments. Although the length is too small, operating expenditures can be high for optical fiber deployment due to dense deployment of small cell BS. So ideally, high data rate is provided by low cost mmWave technology. The mmWave can enable high data rate and low latency connectivity between vehicles and also provides high speed communication between vehicles and cloud for data processing and storage. This can support the mmWave-based vehicle to infrastructure communication. The mmWave can be employed in high speed systems such as mobile phones and virtual reality headsets. There are many challenges for the mmWave MIMO systems and several differences exist between different mmWave MIMO networks. Sub $6 \mathrm{GHz}$ places new constraints on hardware. The hardware challenge at the mmWave are high frequency carrier and bandwidth of the communication channel. One more hardware challenge at mmWave is a large array at both the source and destination due to small wavelength. So large number of analog to digital converters (ADCs) are required by the high number of antennas. By increasing the resolution of the ADCs, the design complexity of the system increases. There are many solutions that have been proposed for mmWave networks such as hybrid beam forming architectures, beam space signal processing and low rate ADC methods [15-17]. The mmWave systems and low frequency systems are having some common properties like multipath delay spread, Doppler shift and angle spread. Also the mmWave has many differences with respect to a low frequency system such as high sensitivity to blockages and strong differences between line of sight (LOS) and non line of sight propagation conditions. High number of antenna arrays at the source and destination makes MIMO signal processing a reality, but this is more challenging at mmWave frequencies due to hardware constraint and different channel characteristics. Thus the novel solutions are needed to overcome hardware constraints, channel modeling and signal processing with large arrays. These will have a significant impact on the design and implementation of the mmWave communication network to maintain active connections with multiple BS's to achieve diversity and overcome blockages. 
Relays and cooperative diversity can enhance coverage and improve signal strength in the mmWave mobile network.

This paper is organized as follows. In section 2, we analyzed the system model for MU-MIMO System. The achievable sum rate is derived for MU-MIMO Systems employing the ZF and MRC receivers with Perfect CSI and imperfect CSI. In section
3, we derived the capacity of optimal precoder/combiner for the mmWave massive MIMO system. Simulation results are shown in section 4 . Section 5 shows the conclusion.

\section{SYSTEM MODEL OF MU-MIMO SYSTEM}

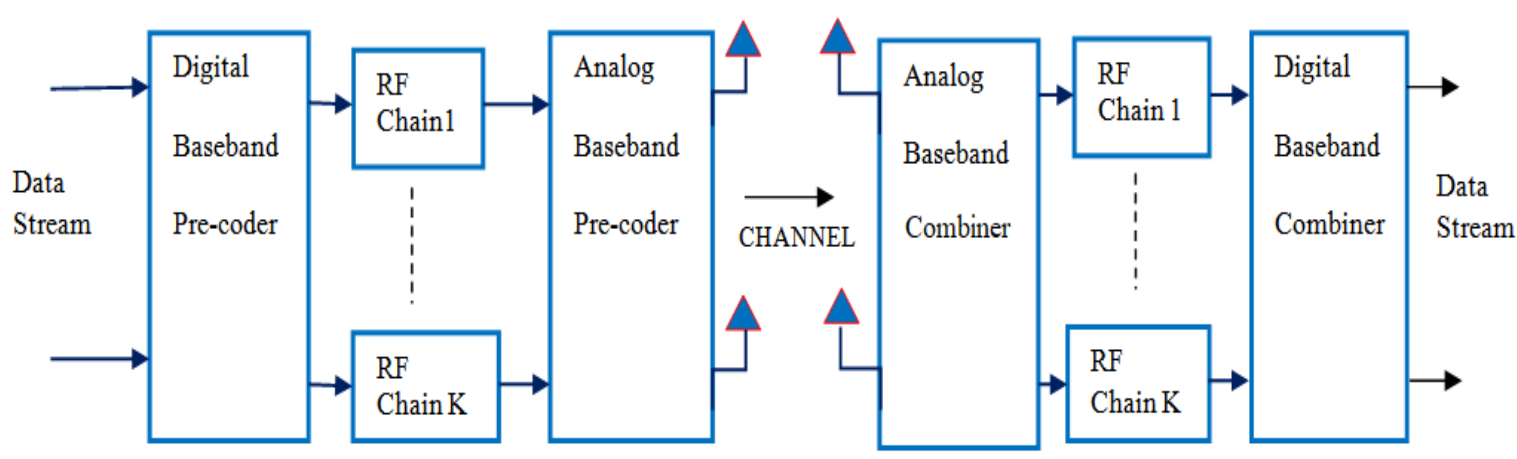

Figure 1. System model of the hybrid mmWave precoder/combiner

\subsection{Perfect CSI for MU-MIMO systems}

The system model of MU-MIMO system can be shown as,

$$
\begin{gathered}
y=\sqrt{P_{U}} \underbrace{\left[\begin{array}{ccccc}
b_{11} & b_{12} & \cdot & \cdot & b_{1 K} \\
b_{21} & b_{22} & \cdot & \cdot & b_{2 K} \\
\cdot & \cdot & \cdot & \cdot & \cdot \\
\cdot & \cdot & \cdot & \cdot & \cdot \\
b_{M 1} & b_{M 2} & \cdot & \cdot & M K
\end{array}\right]}_{B} \underbrace{\left[\begin{array}{c}
x_{1} \\
x_{2} \\
\cdot \\
\cdot \\
x_{K}
\end{array}\right]}_{x}+\underbrace{\left[\begin{array}{c}
n_{1} \\
n_{2} \\
\cdot \\
\cdot \\
n_{M}
\end{array}\right]}_{n} \\
y=\sqrt{P_{U}} B x+n
\end{gathered}
$$

where $P_{U}$ denotes the average transmitted power of each user. $B$ represents the $M \times K$ channel matrix between the BS and $K$ users and models fast fading, geometric attenuation and log-normal shadow fading. $1^{s t}, 2^{\text {nd }} \ldots \ldots . M^{\text {th }}$ row of the $B$ matrix is shown as $b_{1}, b_{2}, \ldots \ldots \ldots b_{M} \cdot b_{m k}$ is the channel coefficient between $\mathrm{m} k^{\text {th }}$ user and $m^{\text {th }}$ antenna from the BS. $x$ is the transmitted data and $n$ is a vector of additive white Gaussian noise with mean zero and variance unity.

The coefficient $b_{m k}$ can be shown as [13],

$$
b_{m k}=h_{m k} \sqrt{\lambda_{k}}
$$

where $h_{m k}$ is the fast fading coefficient between $k^{\text {th }}$ user and $m^{\text {th }}$ antenna of BS. $\lambda_{k}$ is modeled as geometric attenuation and shadow fading.

$$
B=H C^{1 / 2}
$$

where $H$ is $M \times K$ matrix having fast fading coefficient between $K$ user and BS, i.e $[H]_{m k}=h_{m k}$ and $C$ is $K \times$ $K$ diagonal matrix, i.e $[C]_{k k}=\lambda_{k}$.

For perfect channel state information

$$
r=A^{H} y
$$

$$
A= \begin{cases}B & \text { for } M R C \\ B\left(B^{H} B\right)^{-1} & \text { for } Z F\end{cases}
$$

After linear detection, the received vector is given by

$$
r=\sqrt{P_{U}} A^{H} B x+A^{H} n
$$

Considering the MU-MIMO system, the received vector $r$ at the BS can be written as,

$$
r=\sqrt{P_{U}} \sum_{i=1}^{K} a_{k}^{H} b_{i} x_{i}+a_{k}^{H} n
$$

By taking user 1 without loss as the desired user,

$$
r=\sqrt{P_{U}} a_{k}^{H} b_{1} x_{1}+\sqrt{P_{U}} \sum_{i=2}^{K} a_{k}^{H} b_{i} x_{i}+a_{k}^{H} n
$$

The signal to interference noise ratio (SINR) can be given as,

$$
\operatorname{SINR}=\frac{P_{U}\left|a_{k}^{H} b_{1}\right|^{2}}{P_{U} \sum_{i=2}^{K}\left|a_{k}^{H} b_{i}\right|^{2}+\left\|a_{k}\right\|^{2}}
$$

The achievable rate for single cell MU-MIMO can be written as,

$$
\begin{gathered}
R_{p, k}= \\
E\left[\log _{2}\left(1+\frac{\frac{e_{U}}{\sqrt{M}}\left|\hat{b}_{k}^{H} \hat{b}_{1}\right|^{2}}{\frac{e_{U}}{\sqrt{M}} \sum_{i=2}^{K}\left|\hat{b}_{k}^{H} \hat{b}_{i}\right|^{2}+\frac{e_{U}}{\sqrt{M}}\left\|\hat{b}_{k}\right\|^{2} \sum_{i=1}^{K} \frac{\lambda_{i}}{\tau P_{U} \lambda_{i}+1}+\left\|\hat{b}_{k}\right\|^{2}}\right)\right]
\end{gathered}
$$




$$
R_{p . k}=E\left[\log _{2}\left(1+\frac{\frac{P_{U}}{M}\left|a_{k}^{H} b_{1}\right|^{2}}{\underset{\rightarrow \rightarrow 0}{\frac{P_{U}}{M} \sum_{i=2}^{K}\left|a_{k}^{H} b_{i}\right|^{2}}+\left\|a_{k}\right\|^{2}}\right)\right]
$$

The rate scaling is given as,

$$
\log _{2}(1+\operatorname{SINR})=\log _{2}\left(1+e_{U} \lambda_{k}\right)
$$

where $e_{U}$ denote the total transmit power.

The achievable rate for very large value of $\mathrm{M}$ can be written as,

$$
R_{p, k}=\mathrm{E}\left[\log _{2}\left(1+e_{U} \lambda_{k}\right)\right]
$$

In case of MRC, $\mathrm{A}=\mathrm{G}$ so $a_{k}=b_{k}$.

$$
R_{p, k}^{M R C}=\mathrm{E}\left[\log _{2}\left(1+\frac{P_{U}\left|b_{k}^{H} b_{1}\right|^{2}}{P_{U} \sum_{i=2}^{K}\left|b_{k}^{H} b_{i}\right|^{2}+\|\left. b_{k}\right|^{2}}\right)\right]
$$

The power of each user is inversely related to the number of antennas, $P_{U}=\frac{e_{U}}{M}$.

$$
R_{p, k}^{M R C C}=\mathrm{E}\left[\log _{2}\left(1+\frac{\frac{e_{U}}{M}\left|b_{k}^{H} b_{1}\right|^{2}}{\left\|b_{k}\right\|^{2}}\right)\right]
$$

When $\mathrm{M}$ is very large,

$$
R_{p, k}^{\text {MRC }}=\mathrm{E}\left[\log _{2}\left(1+\frac{\frac{e_{U}}{M}\left|b_{k}^{H} b_{1}\right|^{2}}{\left\|b_{k}\right\|^{2}}\right)\right]
$$

By using inequality, $R_{p, k}^{M R C} \geq \hat{R}_{p, k}^{M R C}$. The lower bound on the achievable rate can be given as,

$$
\hat{R}_{p, k}^{M R C}=\log _{2}\left(1+\frac{P_{U}(M-1) \lambda_{k}}{P_{U} \sum_{i=1}^{K} \lambda_{i}+1}\right)
$$

When, $P_{U}=\frac{e_{U}}{M}$, then

$$
\begin{aligned}
& \hat{R}_{p, k}^{M R C}=\log _{2}\left(1+\frac{P_{U}(M-1) \lambda_{k}}{P_{U} \sum_{i=1}^{K} \lambda_{i}+1}\right) \\
& R_{i p, k}=E\left[\log _{2}\left(1+\frac{P_{U}\left|\left(\left(\hat{b}_{k}^{H} \hat{b}_{k}\right)^{-1}\right)^{H} \hat{b}_{k}\right|^{2}}{P_{U} \Sigma_{i=1, i \neq k}^{K}\left|\left(\left(\hat{b}_{k}^{H} \hat{b}_{k}\right)^{-1}\right)^{H} \hat{b}_{i}\right|^{2}+P_{U}\left\|\left(\hat{b}_{k}^{H} \hat{b}_{k}\right)^{-1}\right\|^{2} \sum_{i=1}^{K} \frac{\lambda_{i}}{T_{U} U_{i}+1}+\left\|\left(\hat{b}_{k}^{H} \hat{b}_{k}\right)^{-1}\right\|^{2}}\right)\right]
\end{aligned}
$$

When $M$ is very large, $M \rightarrow \infty$

$$
\hat{R}_{p, k}^{M R C}=\log _{2}\left(1+\lambda_{k} e_{U}\right)
$$

In case of ZF, $A=B\left(B^{H} B\right)^{-1}$ so $a_{k}=\left(b_{k}^{H} b_{k}\right)^{-1}$.

The Achievable sum rate for the ZF receiver can be given as,

$$
\begin{aligned}
& R_{p, k}^{Z F}=\mathrm{E}\left[\log _{2}\left(1+\frac{P_{U}\left|\left(\left(b_{k}^{H} b_{k}\right)^{-1}\right)^{H} b_{1}\right|^{2}}{P_{U} \sum_{i=2}^{K}\left|\left(\left(b_{k}^{H} b_{k}\right)^{-1}\right)^{H} b_{i}\right|^{2}+\left\|\left(b_{k}^{H} b_{k}\right)^{-1}\right\|^{2}}\right)\right] \\
& R_{p, k}^{Z F}=E\left[\log _{2}\left(1+\frac{\frac{e_{U}}{M}\left|\left(\left(b_{k}^{H} b_{k}\right)^{-1}\right)^{H} b_{1}\right|^{2}}{\underbrace{\frac{e_{U}}{M} \sum_{i=2}^{K}\left|\left(\left(b_{k}^{H} b_{k}\right)^{-1}\right)^{H} b_{i}\right|^{2}}_{\rightarrow 0}+\left\|\left(b_{k}^{H} b_{k}\right)^{-1}\right\|^{2}}\right)\right] \\
& R_{p, k}^{Z F}=\mathrm{E}\left[\log _{2}\left(1+\frac{\frac{e_{U}}{M}\left|\left(\left(b_{k}^{H} b_{k}\right)^{-1}\right)^{H} b_{1}\right|^{2}}{\left\|\left(b_{k}^{H} b_{k}\right)^{-1}\right\|^{2}}\right)\right]
\end{aligned}
$$

By using inequality, $R_{p, k}^{Z F} \geq \hat{R}_{p, k}^{Z F}$. The lower bound on the achievable rate can be given as,

$$
\hat{R}_{p, k}^{Z F}=\log _{2}\left(1+P_{U}(M-K) \lambda_{k}\right)
$$

when $P_{U}=\frac{e_{U}}{M}$, then

$$
\hat{R}_{p, k}^{Z F}=\log _{2}\left(1+\frac{e_{U}}{M}(M-K) \lambda_{k}\right)
$$

When $\mathrm{M}$ is very large, $M \rightarrow \infty$

$$
\hat{R}_{p, k}^{Z F}=\log _{2}\left(1+e_{U} \lambda_{k}\right)
$$

\subsection{Imperfect CSI for MU-MIMO systems}

In case of imperfect CSI, the signal to interference noise ratio can be given as [13],

$$
\operatorname{SINR}=\frac{P_{U}\left|\hat{a}_{k}^{H} \hat{b}_{k}\right|^{2}}{\left.P_{U} \sum_{i=1, i \neq k}^{K}||_{k}^{H} \hat{b}_{i}\right|^{2}+P_{U}\left\|\hat{a}_{k}\right\|^{2} \sum_{i=1}^{K} \frac{\lambda_{i}}{\tau P_{U} \lambda_{i}+1}+\left\|\hat{a}_{k}\right\|^{2}}
$$

The Achievable sum rate can be given as,

In case of MRC, $\mathrm{A}=\mathrm{G}$ so $\hat{a}_{k}=\hat{b}_{k}$. 


$$
\begin{aligned}
R_{i p, k}^{M R C} & =E\left[\log _{2}\left(1+\frac{P_{U}\left|\hat{b}_{k}^{H} \hat{b}_{k}\right|^{2}}{P_{U} \sum_{i=1, j \neq k}^{K}\left|\hat{b}_{k}^{H} \hat{b}_{i}\right|^{2}+P_{U}\left\|\hat{b}_{k}\right\|^{2} \sum_{i=1 \frac{1}{\tau P_{U} \lambda_{i}+1}+\left\|\hat{b}_{k}\right\|^{2}}^{2}}\right)\right] \\
R_{i p, k}^{M R C} & =E\left[\log _{2}\left(1+\frac{\lambda_{U}\left|\hat{b}_{k}\right|^{4}}{P_{U} \sum_{i=1, j \neq k}^{K}\left|\hat{b}_{k}^{H} \hat{b}_{i}\right|^{2}+P_{U}\left\|\hat{b}_{k}\right\|^{2} \sum_{i=1}^{K} \frac{\lambda_{i}}{\tau P_{U} \lambda_{i}+1}+\left\|\hat{b}_{k}\right\|^{2}}\right)\right]
\end{aligned}
$$

Put, $P_{U}=\frac{e_{U}}{\sqrt{M}}$

$$
R_{i p, k}^{M R C}=E\left[\log _{2}\left(1+\frac{\frac{e_{U}}{\sqrt{M}}\left|\hat{b}_{k}^{H} \hat{b}_{1}\right|^{2}}{\frac{e_{U}}{\sqrt{M}} \sum_{i=2}^{K}\left|\hat{b}_{k}^{H} \hat{b}_{i}\right|^{2}+\frac{e_{U}}{\sqrt{M}}\left\|\hat{b}_{k}\right\|^{2} \sum_{i=1}^{K} \frac{\lambda_{i}}{\tau P_{U} \lambda_{i}+1}+\left\|\hat{b}_{k}\right\|^{2}}\right)\right]
$$

The achievable uplink rate of the $k^{\text {th }}$ user for the MRC receiver using imperfect CSI, Rayleigh fading and $M>K+$ 1 is bounded as,

Let $P_{U}=\frac{e_{U}}{\sqrt{M}}$, then

$$
\hat{R}_{i p, k}^{M R C}=\log _{2}\left(1+\frac{\tau \frac{e_{U}}{\sqrt{M}}(M-1) \lambda_{k}^{2}}{\left(\tau \frac{e_{U}}{\sqrt{M}} \lambda_{k}+1\right) \sum_{i=1, i \neq k}^{K} \lambda_{i}+(\tau+1) \lambda_{k}+\frac{1}{\frac{e_{U}}{\sqrt{M}}}}\right)
$$

When $M$ is very large, $M \rightarrow \infty$

$$
\hat{R}_{i p, k}^{M R C}=\log _{2}\left(1+\tau \lambda_{k}^{2} e_{U}^{2}\right)
$$

In case of ZF, $\hat{A}=\hat{B}\left(\hat{B}^{H} \hat{B}\right)^{-1}$ so $\hat{a}_{k}=\left(\hat{b}_{k}^{H} \hat{b}_{k}\right)^{-1}$.

$$
\begin{gathered}
R_{i p, k}^{Z F}=E\left[\log _{2}(1+\right. \\
\left.\left.\frac{P_{U}\left|\left(\left(\hat{b}_{k}^{H} \hat{b}_{k}\right)^{-1}\right)^{H} \hat{b}_{k}\right|^{2}}{P_{U} \sum_{i=1, i \neq k}^{K}\left|\left(\left(\hat{b}_{k}^{H} \hat{b}_{k}\right)^{-1}\right)^{H} \hat{b}_{i}\right|^{2}+P_{U}\left\|\left(\hat{b}_{k}^{H} \hat{b}_{k}\right)^{-1}\right\|^{2} \sum_{i=1}^{K} \frac{\lambda_{i}}{\tau P_{U} \lambda_{i}+1}+\left\|\left(\hat{b}_{k}^{H} \hat{b}_{k}\right)^{-1}\right\|^{2}}\right)\right]
\end{gathered}
$$

The achievable uplink rate of the $k^{\text {th }}$ user for the ZF receiver using imperfect CSI, Rayleigh fading and $M>K+$ 1 is bounded as,

$$
\hat{R}_{i p, k}^{Z F}=\log _{2}\left(1+\frac{\tau P_{U}^{2} \lambda_{k}^{2}}{\left(\tau P_{U} \lambda_{k}+1\right) \sum_{i=1}^{K} \frac{P_{U} \lambda_{i}}{\tau P_{U} \lambda_{i}+1}+\tau P_{U} \lambda_{i}+1}\right)
$$

$$
\text { As } P_{U}=\frac{e_{U}}{\sqrt{M}}
$$

$$
\hat{R}_{i p, k}^{Z F}=\log _{2}\left(1+\frac{\tau \frac{e_{U}^{2}}{M} \lambda_{k}^{2}}{\left(\tau \frac{e_{U}}{\sqrt{M}} \lambda_{k}+1\right) \sum_{i=1}^{K} \frac{\frac{e_{U}}{\sqrt{M}} \lambda_{i}}{\tau \frac{e_{U}}{\sqrt{M}} \lambda_{i}+1}+\tau \frac{e_{U}}{\sqrt{M}} \lambda_{i}+1}\right)
$$

When $M$ is very large, $M \rightarrow \infty$.

$$
\hat{R}_{i p, k}^{Z F}=\log _{2}\left(1+\tau \lambda_{k}^{2} e_{U}^{2}\right)
$$

\section{OPTIMAL PRECODER/COMBINER FOR mmWAVE MIMO SYSTEM}

Received matrix $Y$ can be given as [16-17],

$$
Y=\sqrt{P} W_{B B}^{H} W_{R F}^{H} H_{n} F_{R F} F_{B B}+\widetilde{N}
$$

where $F_{R F}=N_{T} \times N_{T}, \quad W_{R F}=N_{R} \times N_{R}, \quad F_{B B}=N_{T} \times$ $N_{T}^{\text {Beam }}$ and $W_{B B}=N_{R} \times N_{R}^{\text {Beam }}$.

Number of RF chains $=N_{R F}$.

The channel model can be written as,

$$
H_{n}=\underbrace{A_{R}}_{N_{R} \times G} \times \underbrace{H}_{G \times G} \times \underbrace{A_{T}^{H}}_{G \times N_{T}}
$$

where $G$ is the number of directional cosine vectors and $H_{n}$ is the sparse combination of directional vector at the transmitter /receiver.

The equivalent sensing matrix is given as,

$$
\begin{gathered}
\operatorname{vec}(Y)=\sqrt{P} Q_{S} \operatorname{vec}(H)+\operatorname{vec}(\widetilde{N}) \\
y=\sqrt{P} Q_{S} h+\tilde{n}
\end{gathered}
$$

Equivalent sensing matrix $Q_{S}=\operatorname{size}_{T}^{\text {Beam }} N_{R}^{\text {Beam }} \times G^{2}$.

$$
Q_{S}=\left(F_{B B}^{T} F_{R F}^{T} A_{T}^{*}\right) \otimes\left(W_{B B}^{H} W_{R F}^{H} A_{R}\right)
$$

The channel estimation problem can be formulated as,

$$
\begin{gathered}
\arg \underbrace{\min }_{h \dashv\left\|y-\sqrt{P} Q_{S} h\right\|_{2}\|y-A h\|_{2}} \\
y=H x+n=H F_{R F} F_{B B} S+n \\
\left(W_{R F}^{o p t}, W_{B B}^{o p t}\right)=\arg \min _{W_{R F}, W_{B B}} E\left\|s-W_{B B}^{H} W_{R F}^{H} y\right\|^{2}
\end{gathered}
$$

where $W_{B B}^{H}=N_{S} \times N_{R F}$ and $W_{R F}^{H}=N_{R F} \times N_{R}$.

The MMSE estimate is shown as,

$$
\begin{gathered}
\hat{s}=W_{M M S E}^{H} y \\
W_{M M S E}^{H}=E\left(s y^{H}\right) E\left(y y^{H}\right)^{-1}=R_{s y} R_{y y}^{-1}
\end{gathered}
$$

Finally MMSE estimate can be written as, 


$$
\hat{s}=\left\|R_{y y}^{1 / 2}\left(W_{M M S E}-W_{R F} W_{B B}\right)\right\|_{F}^{2}
$$

MMSE minimization can be given as,

$$
\left(W_{R F}^{o p t}, W_{B B}^{o p t}\right)=\arg \min _{W_{R F}, W_{B B}} E\left\|R_{y y}^{1 / 2}\left(W_{M M S E}-W_{R F} W_{B B}\right)\right\|_{F}^{2}
$$

The capacity can be given as,

$$
C=B \log _{2}(1+\operatorname{SINR})
$$

\section{SIMULATION RESULTS}

Figure 2 shows that there is a much improvement in the uplink sum rate for the $\mathrm{ZF}$ receiver than MRC receiver with perfect channel state information in the massive MIMO system. The uplink sum rate also increases as the number of base station antenna increases. In figure 3, comparison of uplink sum rate for varying number of users is done. The uplink sum rate is higher as the number of users increases with imperfect channel state information in the massive MIMO system. In figure 4, it is shown that as the number of transmit and receive antenna increases, the capacity of the mmWave massive MIMO system increases. Capacity of hybrid MIMO outperforms conventional MIMO system. Figure 5 shows an improvement in the capacity of the system as the number of radio frequency chain increases.

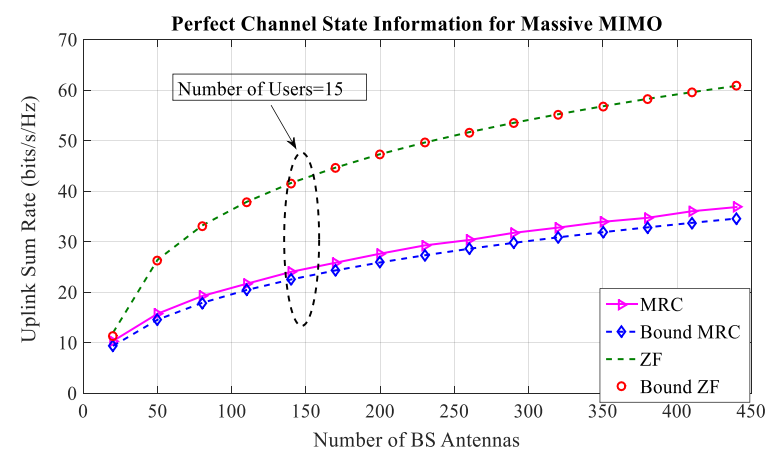

Figure 2. Numerically evaluated values and lower bounds of uplink sum rate for different number of base station antenna for MRC and ZF receiver with with perfect channel state information for $\mathrm{K}=15$ (Number of Users)

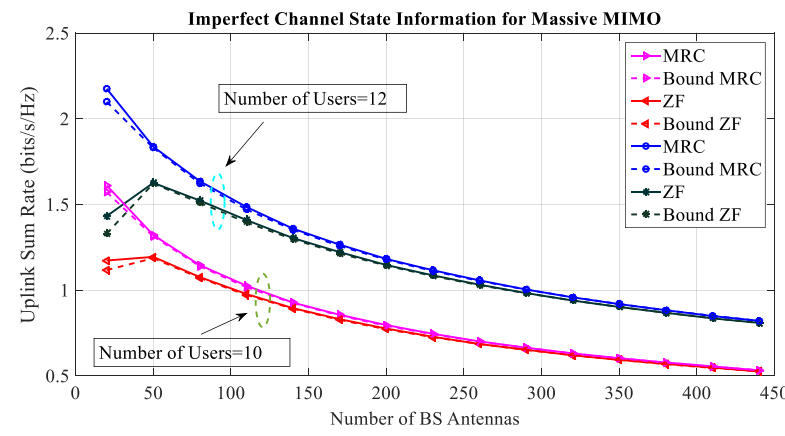

Figure 3. Comparison of numerically evaluated values and lower bounds of uplink sum rate for different number of base station antenna for MRC and ZF receiver with imperfect channel state information for $\mathrm{K}=10$ (Number of Users) and $\mathrm{K}=12$ (Number of Users)

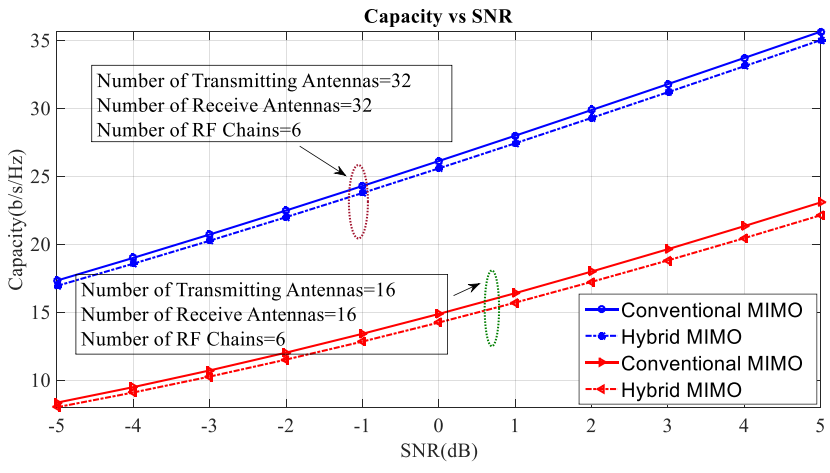

Figure 4. Comparision of Capacity versus signal to noise ratio for Conventional MIMO and Hybrid MIMO with $T x=$ 32, $R x=32, n R F=6$ and $T x=16, R x=16, n R F=6$

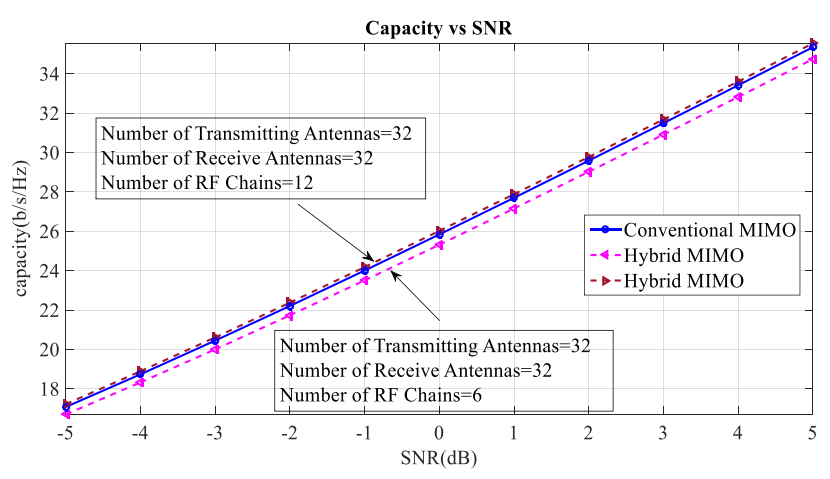

Figure 5. Comparison of Capacity versus signal to noise ratio for Conventional MIMO and Hybrid MIMO with $T x=$ $32, R x=32, n R F=12$ and $T x=32, R x=32, n R F=6$

\section{CONCLUSION}

Massive MIMO systems offer an improvement in the uplink sum rate with increasing the number of base station antennas. $\mathrm{ZF}$ forcing receiver outperforms the MRC with perfect CSI and Imperfect CSI. With imperfect CSI, as the number of users increases the uplink sum rate also increases. In the mmWave massive MIMO system, there is an improvement in channel capacity as the number of transmit and receive antenna increases. Further, as the number of the RF chains decreases, the capacity of the mmWave massive MIMO system with MMSE receiver also degrades.

\section{REFERENCES}

[1] Magrini, A., Lazzari, S., Marenco, L., Guazzi, G. (2017). A procedure to evaluate the most suitable integrated solutions for increasing energy performance of the building's envelope, avoiding moisture problems. International Journal of Heat and Technology, 35(4): 689-699. https://doi.org/10.18280/ijht.350401

[2] Rappaport, T.S., Sun, S., Mayzus, R., Zhao, H., Azar, Y., Wang, K., Wong, G.N., Schulz, J.K., Samimi, M., Gutierrez, F. (2013). Millimeter wave mobile communications for $5 \mathrm{G}$ cellular: It will work! In IEEE Access, 1: 335-349. http://dx.doi.org/10.1109/ACCESS.2013.2260813

[3] Andrews, J.G., Bai, T., Kulkarni, M.N., Alkhateeb, A., Gupta, A.K., Heath, R.W. (2017). Modeling and 
analyzing millimeter wave cellular systems. In IEEE Transactions on Communications, 65(1): 403-430. http://dx.doi.org/10.1109/TCOMM.2016.2618794

[4] MacCartney, G.R., Samimi, M.K., Rappaport, T.S (2015). Exploiting directionality for millimeter-wave wireless system improvement. 2015 IEEE International Conference on Communications (ICC), 2416-2422. http://dx.doi.org/10.1109/ICC.2015.7248687

[5] Sachan, V., Shankar, R., Mishra, R.K. (2017). Selective Decode-forward cooperative communication over Nakagami-m fading channel with channel estimation error. Journal of Telecommunication, Electronic and Computer Engineering (JTEC), 9(2-6): 85-90.

[6] Sachan, V., Kumar, I., Shankar, R., Mishra, R.K. (2018). Analysis of transmit antenna selection based selective decode forward cooperative communication protocol. Traitement du Signal, 35(1): 47-60. http://dx.doi.org/10.3166/TS.35

[7] Sachan, V., Shankar, R., Kumar, I., Mishra, R.K. (2019). Performance analysis of SM-MIMO system employing binary PSK and M'ary PSK techniques over different fading channels. Procedia Computer Science, 152: 323 332. http://dx.doi.org/10.1016/j.procs.2019.05.010

[8] Shankar, R., Kumar, G., Sachan, V., Mishra, R.K. (2018). An investigation of two phase multi-relay S-DF cooperative wireless network over time-variant fading channels with incorrect CSI. Procedia Computer Science, 125:

871-879. http://dx.doi.org/10.1016/j.procs.2017.12.111

[9] Ngo, H.Q., Larsson, E.G., Marzetta, T.L. (2013). Energy and spectral efficiency of very large multiuser MIMO systems. In IEEE Transactions on Communications, 61(4): 1436-1449. http://dx.doi.org/10.1109/TCOMM.2013.020413.11084 8

[10] Hoydis, J., ten Brink, S., Debbah, M. (2013). Massive MIMO in the UL/DL of cellular networks: How many antennas do we need? IEEE Journal on Selected Areas in Communications, 31(2): 160-171. http://dx.doi.org/10.1109/JSAC.2013.130205

[11] Yang, H., Marzetta, T.L. (2013). Performance of conjugate and zeroforcing beamforming in large-scale antenna systems. IEEE Journal on Selected Areas in Communications, $31(2)$ : $172-179$ http://dx.doi.org/10.1109/JSAC.2013.130206

[12] Rusek, F., Persson, D., Lau, B.K., Larsson, E.G., Marzetta, T.L., Edfors, O., Tufvesson, F. (2013). Scaling up MIMO: Opportunities and challenges with very large arrays. IEEE Signal Processing Magazine, 30(1): 40-60. http://dx.doi.org/10.1109/MSP.2011.2178495
[13] Ngo, H.Q., Larsson, E.G., Marzetta, T.L. (2013). The multicell multiuser MIMO uplink with very large antenna arrays and a finite-dimensional channel. In IEEE Transactions on Communications, 61(6): 2350-2361. http://dx.doi.org/10.1109/TCOMM.2013.032713.12040 8

[14] Kumar, I., Sachan, V., Shankar, R., Mishra, R.K. (2018). An investigation of wireless S-DF hybrid satellite terrestrial relaying network over time selective fading channel. Traitement du Signal, 35(2): 103-120. http://dx.doi.org/10.3166/ts.35.103-120

[15] Jose, J., Ashikhmin, A., Marzetta, T.L., Vishwanath, S. (2011). Pilot contamination and precoding in Multi-Cell TDD systems. In IEEE Transactions on Wireless Communications, $\quad 10(8)$ : 2640-2651. http://dx.doi.org/10.1109/TWC.2011.060711.101155

[16] Ayach, O.E., Rajagopal, S., Abu-Surra, S., Pi, Z., Heath, R.W. (2014). Spatially sparse precoding in millimeter wave MIMO systems. In IEEE Transactions on Wireless Communications, 13(3): $1499-1513$ http://dx.doi.org/10.1109/TWC.2014.011714.130846

[17] Alkhateeb, A., El Ayach, O., Leus, G., Heath, R.W. (2014). Channel estimation and hybrid precoding for millimeter wave cellular systems. In IEEE Journal of Selected Topics in Signal Processing, 8(5): 831-846. http://dx.doi.org/10.1109/JSTSP.2014.2334278

\section{NOMENCLATURE}

channel capacity

total transmitted power

single antenna user

Base station antenna

additive white Gaussian noise

number of radio frequency chain

average transmitted power

transmitted data

received vector

achieved sum rate

\section{Greek symbols}

$\lambda$

$\tau$

geometric distribution

length of pilot sequence

\section{Subscripts}

u 